\title{
Pore-Forming M2 Domains of CHRNA4 and CHRNB2 Mutations In Autosomal Dominant Nocturnal Frontal Lobe Epilepsy (ADNFLE) Phenotype
}

\author{
Behzad Saberi* and Behrouz Saberi Tehrani \\ Medical Research, Iran \\ *Corresponding author: Behzad Saberi, Medical Research, 45, Sha. Karimi, Nikbakht Str., 8164717845, Esfahan, Iran. \\ To Cite This Article: Behzad Saberi, Behrouz Saberi. Pore-Forming M2 Domains of CHRNA4 and CHRNB2 Mutations In Autosomal Dominant \\ Nocturnal Frontal Lobe Epilepsy (ADNFLE) Phenotype. Am J Biomed Sci \& Res. 2019 - 1(5). AJBSR.MS.ID.000547. \\ DOI: 10.34297/AJBSR.2019.01.000547
}

Received: February 27, 2019 | Published: March 08, 2019

\section{Introduction}

Autosomal Dominant Nocturnal Frontal Lobe Epilepsy (ADNFLE) is an uncommon form of epilepsy which causes partial seizures that usually occur at night and almost exclusively during sleep which are characterized by brief tonic or hyperkinetic motor activity without alteration in consciousness. There is some specific cholinergic receptor, nicotinic genes which their mutations can cause ADNFLE like CHRNA2, CHRNA4 and CHRNB2. In this study we have reviewed the effects of Pore-forming M2 domains of CHRNA4 and CHRNB2 genes in occurrence of ADNFLE.

Autosomal Dominant Nocturnal Frontal Lobe Epilepsy (ADNFLE) is an uncommon form of epilepsy which its seizure characteristics tend to occur in clusters - brief motor episodes with hyperkinetic and dystonic features or tonic manifestations. ADNFLE as a focal epilepsy syndrome is described with a single gene inheritance pattern. Its onset ranges from infancy to adulthood although late childhood - about seven to twelve years of age - is the main onset time. Usually the first seizure occurs before the age of twenty and the men and women can be equally affected. The seizures in ADNFLE are usually of sudden onset and would terminate without Post-ictal symptoms and Consciousness is usually preserved during the seizures. A non-specific Aura can be experienced in about two thirds of the patients. Occurrence of secondary GTCSs is very infrequent in ADNFLE unless in the untreated patients or after medication withdrawal. Severity of ADNFLE is markedly variable between families and their affected members [1-5].

Penetrance of ADNFLE is about seventy percent in some families and can be very much lower in others. CHRNA4 and CHRNB2 are two ADNFLE gene loci which were initially identified. CHRNA4 encodes the neuronal nicotinic acetylcholine receptor Alpha 4-subunit protein and is located at chromosome region 20q13.2q13.3. CHRNB2 encodes the neuronal nicotinic acetylcholine receptor Beta 2-subunit protein and is located at chromosome region 1q21.3 (Later in some families with ADNFLE such genes were excluded, and one family was linked to chromosome 15q24). CHRNA2 is another identified gene for a type of ADNFLE which a familial sleep-related focal epilepsy is and encodes the neuronal cholinergic receptor Alpha 2- subunit protein and is located on chromosome 8p21 [6,7].

Acetylcholine signals can be transduced by two different types of receptors which are Muscarinic and Nicotinic. Muscarinic receptors signals are relatively slow but Nicotinic receptors mediate fast synaptic transmission. They are very important for development and synaptic plasticity and participate in learning, memory, and attention. Acetylcholine is released into the synaptic cleft but also at non-synaptic sites.

Its Release is episodic and in a pulsed manner. Nicotinic acetylcholine receptors can adopt one of three functional states based on Presence, Abundance and Timing of acetylcholine binding: Closed at rest, Open pore and Closed desensitized. Any Prolonged exposure to low doses of acetylcholine, nicotine or a nicotinic agonist can cause desensitization which can stabilize the receptor in a closed state and unresponsive to further stimulation. Five transmembrane subunits which are arranged around a central water-filled pore would assemble Nicotinic acetylcholine receptors. Missense or Insertions-deletions mutations in CHRNA4, CHRNB2 and CHRNA2 which affect the transmembrane or nearby domains of the respective proteins and consequently affecting the kinetics of the ion pore, can cause ADNFLE. Mutations in CHRNB2 and CHRNA2 are usually Gain-of-function ones but CHRNA4 mutations can be either Gain-of-function or Loss-of-function ones. The mutations can convert a Serine to Phenylalanine in the M2 transmembrane domain which is an important structure which mediates Ionic permeability and also affect Pore-forming amino acids and cause ADNFLE [8-10]. 


\section{References}

1. Scheffer IE, Bhatia KP, Lopes-Cendes I, Fish DR, Marsden CD, et al. (1995) Autosomal dominant nocturnal frontal lobe epilepsy. A distinctive clinical disorder. Brain 118: 61-73.

2. Bi Corcia G, Blasetti A, De SM, Verrotti A, Chiarelli F (2005) Recent advances on autosomal dominant nocturnal frontal lobe epilepsy: "understanding the nicotinic acetylcholine receptor (nAChR)". Eur J Paediatr Neurol 9(2): 59-66.

3. McLellan A, Phillips HA, Rittey C, Kirkpatrick M, Mulley JC, et al. (2003) Phenotypic comparison of two Scottish families with mutations in different genes causing autosomal dominant nocturnal frontal lobe epilepsy. Epilepsia 44(4): 613-617.

4. Scheffer IE, Bhatia KP, Lopes-Cendes I, Fish DR, Marsden CD, et al. (1994) Autosomal dominant frontal epilepsy misdiagnosed as sleep disorder. Lancet 343(8896): 515-517.

5. Brodtkorb E, Picard F (2006) Tobacco habits modulate autosomal dominant nocturnal frontal lobe epilepsy. Epilepsy Behav 9(3): 515-520.
6. Berkovic SF, Scheffer IE. Autosomal dominant nocturnal frontal lobe epilepsy.

7. Duga S, Asselta R, Bonati MT, Malcovati M, Dalpra L, et al. (2002) Mutational analysis of nicotinic acetylcholine receptor beta2 subunit gene (CHRNB2) in a representative cohort of Italian probands affected by autosomal dominant nocturnal frontal lobe epilepsy. Epilepsia 43(4): 362-364.

8. Ito M, Kobayashi K, Fujii T, Okuno T, Hirose S et al. (2000) Electroclinical picture of autosomal dominant nocturnal frontal lobe epilepsy in a Japanese family. Epilepsia 41(1): 52-58.

9. Oldani A, Zucconi M, Asselta R, Modugno M, Bonati MT, et al. (1998) Autosomal dominant nocturnal frontal lobe epilepsy. A videopolysomnographic and genetic appraisal of 40 patients and delineation of the epileptic syndrome. Brain 121(Pt 2): 205-223.

10. Combi R, Dalpra L, Tenchini ML, Ferini-Strambi L (2004) Autosomal dominant nocturnal frontal lobe epilepsy - a critical overview. J Neurol 251(8): 923-934 